\title{
Dinâmica sazonal da produção e decomposição de serrapilheira em floresta tropical de transição
}

\author{
Luciana Sanches ${ }^{1}$, Carla M. A. Valentini ${ }^{2}$, Marcelo S. Biudes ${ }^{1}$ \& José de S. Nogueira ${ }^{1}$
}

\begin{abstract}
RESUMO
Em função de se analisar a produção, dinâmica e decomposição de serrapilheira em busca da sazonalidade do microclima em uma floresta tropical de transição Amazônia Cerrado, propõe-se estimar a produção de serrapilheira, por meio de caixas de coleta de $1 \mathrm{~m}^{2}$ e o acúmulo de serrapilheira sobre o solo por meio de quadrantes de $25 \mathrm{x} 25 \mathrm{~cm}$, distribuídos aleatoriamente. Determinaram-se a constante de decaimento $(K)$, o coeficiente de retorno de serrapilheira $\left(K_{L}\right)$ e a constante de decomposição $\left(K^{\prime}\right)$. Com o emprego de bolsas de nylon preenchidas com folhas de espécies de relevância na floresta (Tovomita schomburgkki e Brosimium lactescens) estudou-se a decomposição de folhas e se monitaram os fatores ambientais por meio de equipamentos instalados em uma torre micrometeorológica. A produção de serrapilheira não só se apresentou de forma sazonal, com significativa influência da umidade relativa do ar e da precipitação, em uma floresta de transição, mas foi majoritariamente composta de folhas seguidas de galhos e miscelânea (flores e frutos). A decomposição de folhas em florestas tropicais foi acelerada em ambas as estações, seca-úmida e úmida-seca, sendo a velocidade de decomposição das folhas maior na estação úmida-seca.
\end{abstract}

Palavras-chave: acúmulo de serrapilheira no solo, Amazônica, decomposição de folhas

\section{Seasonal dynamics of the litterfall production and decomposition in tropical transitional forest}

\begin{abstract}
The objective of this study was to analyze the production, dynamics and litter decomposition as a function of the seasonality of the microclimate in a transitional tropical forest of Amazonia Cerrado. Estimate of litterfall was carried out with collection boxes of $1 \mathrm{~m}^{2}$ and floor forest mass with quadrants of $25 \times 25 \mathrm{~cm}$ distributed randomly. The decline constant (K), litter turnover rate $\left(\mathrm{K}_{\mathrm{L}}\right)$ and litter decomposition rate $\left(\mathrm{K}^{\prime}\right)$ were determined by estimation of litterfall and forest floor litter mass. Leaf decomposition was studied by litter bags with leaves of species of relevance (Tovomita schomburgkki and Brosimium lactescens) in the forest. The analysis of the environmental factors was measured through micrometeorological equipments. The litterfall was seasonal in nature, with significant influence from relative air humidity and rainfall in a transitional forest. Litterfall was mainly composed of leaves, followed by twigs, and miscellany (flowers and fruits). The leaf decomposition in tropical forests was accelerated in seasons, dry-wet and wet-dry, being the leaf decomposition higher in the wet-dry season.
\end{abstract}

Key words: forest floor litter mass, Amazon, leaf decomposition

1 Pós-graduação em Física Ambiental, UFMT. Av. Fernando Corrêa s/n, CEP 78060-90, Cuiabá, MT. Fone: (65) 3615-8748. E-mail: Isanches@ufmt.br

${ }^{2}$ Centro Federal de Educação Tecnológica de Cuiabá. Rua Professora Zulmira Canavarros, 95, CEP 78005-200, Cuiabá, MT. 


\section{INTRODUÇÃO}

A serrapilheira representa o maior caminho biológico da transferência de elementos da vegetação para o solo (Xu \& Hirata, 2002); ela e seu processo de decomposição mantêm os nutrientes no solo, influenciam a produção primária e regulam o fluxo de energia e os ciclos de nutrientes em ecossistemas florestais (Waring \& Schlesinger, 1985). Os principais fatores que agem na decomposição da serrapilheira, são clima, qualidade de serrapilheira e natureza e abundância de organismos decompositores (Coûteaux et al., 1995; Smith \& Bradford, 2003). Estudos avaliam o papel da serrapilheira (Aerts, 1997) ou dos organismos do solo (Heneghan et al., 1999) como fatores determinantes na sua decomposição.

A temperatura e umidade são os principais fatores que afetam o processo de decomposição (Lavelle et al., 1993) e suas variações podem abalar o comportamento dos sistemas atmosféricos, visto que essas variáveis se interagem ao mesmo tempo. Na Amazônia central existem evidências de que as taxas de decomposição são muito afetadas pelas variações sazonais, formando padrões distintos nas estações chuvosa e seca (Cornu et al., 1997).

As florestas tropicais possuem uma comunidade de múltiplas espécies de flora, fornecendo à serrapilheira composição diversificada e um ciclo de nutriente mais estável estabelecendo, conseqüentemente, uma comunidade de decompositores diversificada e de intensa dinâmica; este fato ocorre em virtude dessas florestas se situarem em uma região em que o clima é definido por uma estação seca e outra úmida.

A natureza e a magnitude dessas estações podem interferir na produção e acúmulo de serrapilheira no solo, valores de retorno e de decomposição de serrapilheira, podendo apresentar diferenças interanuais (Wieder \& Wright, 1995). Esta variabilidade anual na produção de serrapilheira enfatiza uma das dificuldades na estimativa da taxa de decomposição pela produção e acúmulo de serrapilheira (Lamb, 1985). Entender a estrutura e a composição do ecossistema florestal é um dos primeiros passos para se determinar um manejo melhor de sistemas. Os estudos de produção de serrapilheira são importantes visto que contribuem para a definição de modelos de fluxo e conteúdo de carbono, em determinadas regiões e mostra, de forma mais consistente, as suas dinâmicas ecossistêmicas.

Este trabalho foi realizado com o objetivo de analisar a produção, dinâmica e decomposição de serrapilheira em função da sazonalidade do microclima em uma floresta tropical de transição Amazônia Cerrado.

\section{MATERIAL E MÉTODOS}

\section{Descrição da área de estudo}

Este estudo foi desenvolvido em uma área localizada a aproximadamente $50 \mathrm{~km} \mathrm{NE}$ da cidade de Sinop, norte de

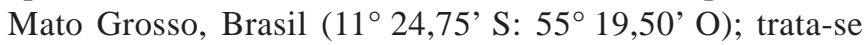
de uma região de transição entre a floresta tropical úmida e o Cerrado, com altura média do dossel da ordem de $30 \mathrm{~m}$, que apresenta sazonalidade característica com estação seca de junho a setembro e uma estação chuvosa de dezembro a fevereiro.

\section{Medidas micrometeorológicas e auxiliares}

Dados micrometeorológicos auxiliares, como temperatura e umidade relativa do ar (medidas com sensor HMP-35, Vaisala, Inc., Helsinki, Finland) e precipitação (medida com pluviômetro tipo “monjolo” Mod. 2501 Sierra-Misco Inc. Berkeley, CA, EUA), instalados a $41 \mathrm{~m}$ de altura em uma torre metálica, foram coletados por um sistema de aquisição de dados (Campbell CR10X - Campbell Scientific) e as médias de 30 min armazenadas em módulos de memória.

\section{Produção de serrapilheira}

Para determinação da produção de serrapilheira usaram-se 20 coletores de $1 \mathrm{~m}^{2}$ cada um, instalados próximos à torre micrometeorológica, com coletas realizadas de dezembro de 2000 a dezembro de 2003; em 2001 as deposições de serrapilheira foram recolhidas mensalmente dos coletores e, a partir de 2002, esta coleta se tornou semanal. Cada amostra coletada foi separada em frações, folhas, galhos, flores e frutos e secada em estufa a $70{ }^{\circ} \mathrm{C}$ durante $72 \mathrm{~h}$, e depois realizadas suas pesagens (precisão $\pm 1 \mathrm{~g}$ ); enfim, para análise das relações entre a umidade relativa, precipitação e temperatura e a produção de serrapilheira, aplicou-se a análise de correlação.

\section{Acúmulo de serrapilheira no solo}

Para determinação do estoque no solo coletaram-se, aleatoriamente, 20 amostras de serrapilheira em quadrantes de 25 x $25 \mathrm{~cm}$ nas proximidades de cada caixa de coleta de serrapilheira; este monitoramento foi realizado mensalmente, de janeiro de 2002 a dezembro de 2003; as amostras coletadas foram peneiradas em malha de 2,0 mm e separadas por folhas, galhos, flores, frutos e raízes; após secagem em estufa sob as mesmas condições descritas anteriormente, realizaram-se as pesagens. Efetuou-se a comparação pareada pelo teste T, das frações de folha com as outras frações componentes da serrapilheira.

\section{Decomposição de serrapilheira}

A decomposição de serrapilheira realizada por períodos, foi estimada pelo modelo exponencial definido por Wieder \& Wright (1995), como constante de decaimento (K) em que se calculou a queda diária e a queda mensal, pelas Eq. 1 e 2 , respectivamente.

$$
\begin{gathered}
\mathrm{LFD}=\frac{\mathrm{LFW}}{\sum_{\mathrm{i}=1}^{7} \mathrm{e}^{-\mathrm{k} \cdot \mathrm{i}}} \\
\mathrm{FFLM}_{\mathrm{x}}=\mathrm{FFLM}_{\mathrm{x}-1} \cdot \mathrm{e}^{-28 \cdot \mathrm{k}}+\sum_{\mathrm{w}=1}^{4} \sum_{\mathrm{i}=1}^{7} \mathrm{LFD}_{\mathrm{w}} \cdot \mathrm{e}^{-\mathrm{k}[29-(7(\mathrm{w}-1)+\mathrm{i}]}
\end{gathered}
$$

em que: LFD é a queda diária $\left(\mathrm{g} \mathrm{m}^{-2} \mathrm{~d}^{-1}\right)$; LFW é a queda semanal ( g m$^{-2}$ semana $^{-1}$ ); K é a constante de decaimento; i é o indexador do dia da semana; $F_{F L M}$ é a massa no solo no mês $\mathrm{x} ; \mathrm{FFLM}_{\mathrm{x}-1}$ é a massa no solo no mês x-1 e $\mathrm{LFD}_{\mathrm{w}}$ é 
a queda diária calculada para a semana de índice w.

$O$ coeficiente de retorno de serrapilheira $\left(K_{L}\right)$ é definido como a produção anual de serrapilheira (LF) dividida pela média de serrapilheira acumulada no solo da floresta (FFM), Eq. 3.

$$
\mathrm{K}_{\mathrm{L}}=\frac{\mathrm{LF}}{\mathrm{FFM}}
$$

Em adição, o modelo de balanço de massa (Reiners \& Reiners, 1970; Xu \& Hirata, 2002; Lodhiyal \& Lodhiyal, 2003) foi utilizado para estimar a constante de decomposição (K'), definida como a produção anual de serrapilheira (LF) dividida pela soma da média de serrapilheira acumulada no solo da floresta (FFM) e a produção anual de serrapilheira (LF), Eq. 4.

$$
\mathrm{K}^{\prime}=\frac{\mathrm{LF}}{\mathrm{FFM}+\mathrm{LF}}
$$

O tempo de retorno (t) de serrapilheira (anos) é o recíproco da taxa de retorno, Eq. 5.

$$
\mathrm{t}=\frac{1}{\mathrm{~K}_{\mathrm{L}}}
$$

\section{Decomposição de folhas}

Em relação ao estudo da dinâmica e taxa de decomposição das folhas, utilizaram-se bolsas de nylon instaladas em vinte pontos de amostragem; em cada um desses pontos foram colocadas nove bolsas de nylon de dimensão de 30 x $30 \mathrm{~cm}$, instaladas em diferentes períodos de transição, a primeira em período seco-úmido (agosto de 2002) e a segunda em período úmido-seco (maio de 2003). A duração dos experimentos foi 129 e 91 dias, depois da instalação de cada experimento, respectivamente; cada bolsa de nylon foi preenchida com um peso seco entre 2 a $5 \mathrm{~g}$ de folhas maduras, de duas espécies dominantes na região, Tovomita schomburgkki e Brosimium lactescens, colhidas na área e secadas sob as condições já descritas e pesadas.

\section{RESULTADOS E DISCUSSÃO}

\section{Variações sazonais na micrometeorologia}

A precipitação acumulada em outubro a abril (período úmido) foi 165 a $484 \mathrm{~mm}, 23$ a $388 \mathrm{~mm}$ e 271 a $600 \mathrm{~mm}$, em 2001, 2002 e 2003, respectivamente; de maio a setembro (período seco) as precipitações se mantiveram entre 0 a 177, 0 a 62, e 25 a 70 mm, nos anos 2001, 2002 e 2003, respectivamente; em junho e julho não ocorreu precipitação. A precipitação acumulada foi 2006, 1861 e $2645 \mathrm{~mm}$ em 2001, 2002 e 2003, respectivamente; outra característica marcante foi a sazonalidade da umidade relativa ocorrendo, em agosto; com médias mínimas de 62,76; 65,94 e 64,9\%, em 2001, 2002 e 2003, respectivamente, e máximas de 88,4 e $85,9 \%$ de janeiro de 2001 e 2003, respectivamente, e 86,56\% em fevereiro de 2001 (Figura 1). A temperatura do
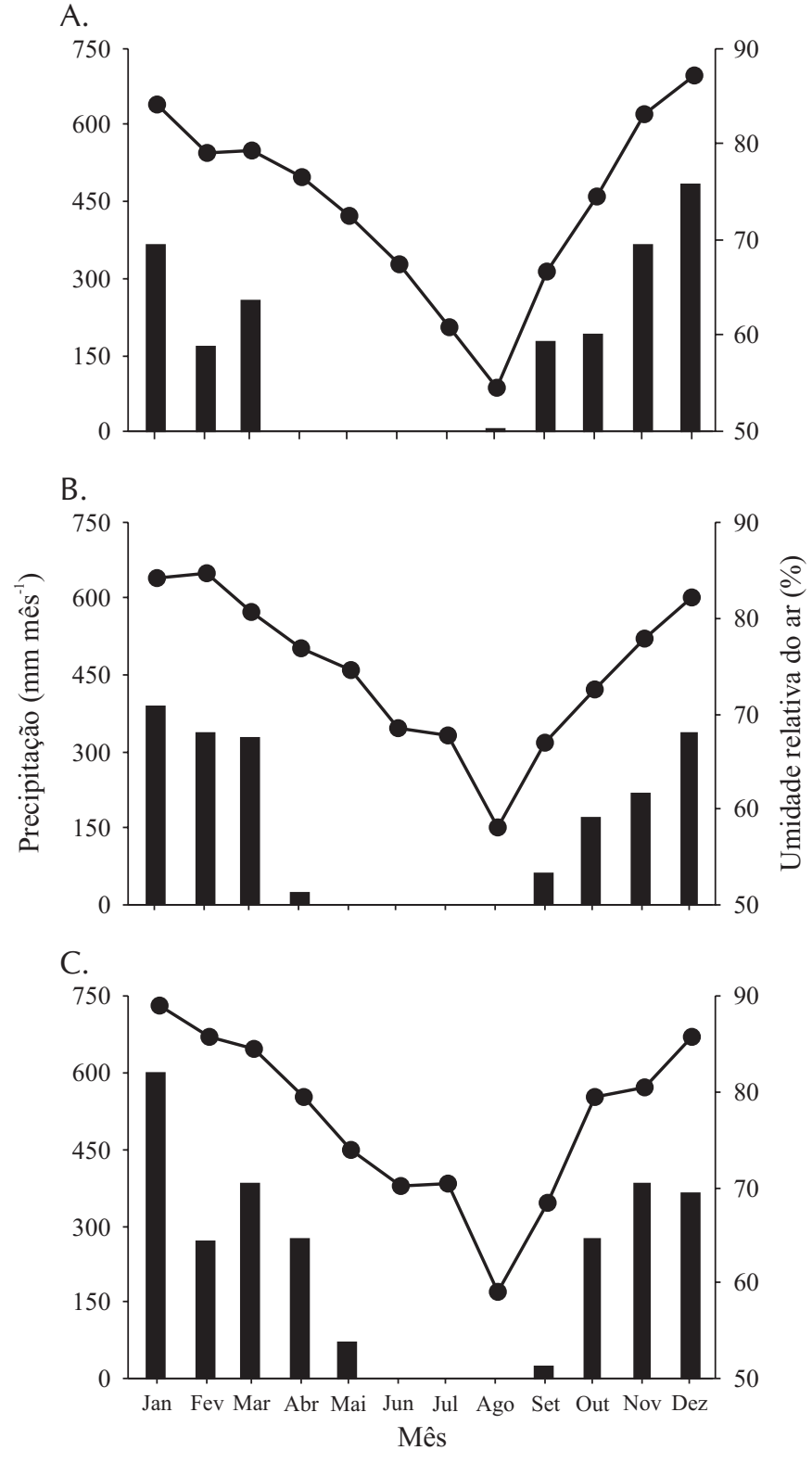

Figura 1. Precipitação mensal e média mensal da umidade relativa do ar, em (A) 2001, (B) 2002 e (C) 2003 na floresta de transição

ar apresentou sazonalidade com médias máximas mensais de 26,6; 30,3 e 26,4 ${ }^{\circ} \mathrm{C}$ em outubro de 2001; novembro de 2002 e dezembro de 2003, respectivamente, além de médias mínimas mensais de 24,5; 24,1 e $23,0^{\circ} \mathrm{C}$, em junho de 2001; maio de 2002 e agosto de 2003, respectivamente (Figura 2).

\section{Produção de serrapilheira}

A produção média mensal de serrapilheira variou de 0,05 a 1,53 t ha mês ${ }^{-1}$ (Tabela 1; Figura 3); os valores apresentados no período seco foram similares aos reportados em uma floresta tropical úmida no Panamá (Wieder \& Wright, 1995) e floresta secundária Nacional Mário Xavier - FLONA, Seropédica, no Rio de Janeiro (Corrêa Neto et al., 2001).

Estimou-se em 2002 e 2003 uma produção anual de folhas de 6,86 e 4,93 $\mathrm{t} \mathrm{ha}^{-1} \mathrm{ano}^{-1}$, respectivamente. Os valores estimados de produção anual foram inferiores aos apresentados em uma floresta natural intacta de pinheiro 


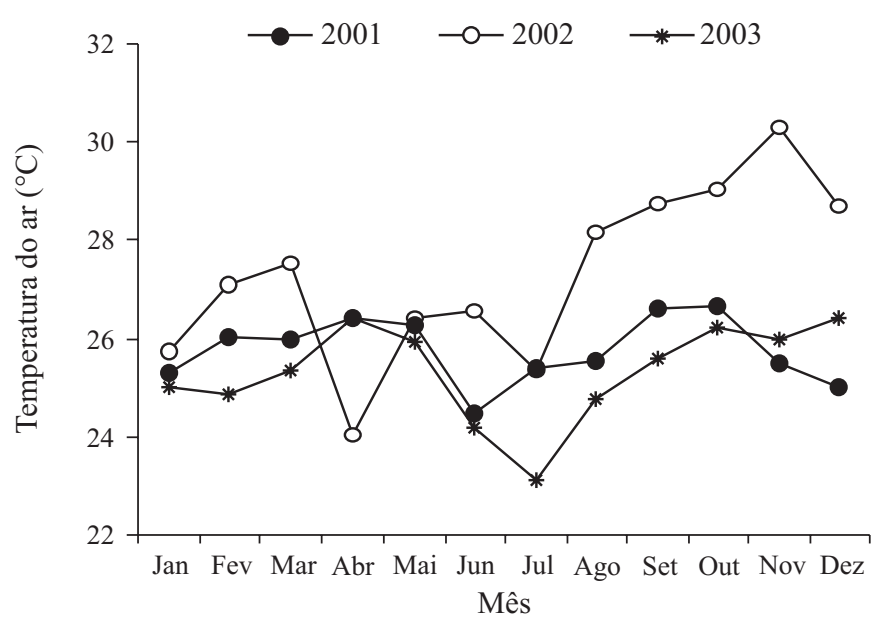

Figura 2. Média mensal da temperatura do ar, em 2001, 2002 e 2003 em uma floresta de transição

Tabela 1. Produção de serrapilheira (t ha- ${ }^{-1} \mathrm{mê}^{-1}$, média $\pm \mathrm{DP}$ ) e acúmulo de serrapilheira no solo (t ha-1 ${ }^{-1}$, média \pm DP) em uma floresta de transição

\begin{tabular}{|c|c|c|c|c|c|}
\hline \multirow{2}{*}{ Mês/Ano } & \multicolumn{4}{|c|}{ Produção de serrapilheira ( $t$ ha ${ }^{-1}$ mês $^{-1}$, média $\left.\pm \mathrm{DP}\right)$} & \multirow{2}{*}{$\begin{array}{l}\text { Acúmulo de } \\
\text { serrapilheira } \\
\text { no solo } \\
\text { (t ha-1 } \\
\text { média } \pm \text { DP) }\end{array}$} \\
\hline & Folhas & Galhos & Miscelânea & Total & \\
\hline Jan./2001 & $0,42 \pm 0,14$ & $0,16 \pm 0,59$ & $0,06 \pm 0,05$ & $0,64 \pm 0,64$ & \\
\hline Fev./2001 & $0,24 \pm 0,07$ & $0,13 \pm 0,22$ & $0,03 \pm 0,07$ & $0,41 \pm 0,26$ & \\
\hline Mar./2001 & $0,43 \pm 0,16$ & $0,15 \pm 0,09$ & $0,05 \pm 0,09$ & $0,63 \pm 0,20$ & \\
\hline Abr./2001 & $0,38 \pm 0,11$ & $0,16 \pm 0,19$ & $0,07 \pm 0,16$ & $0,61 \pm 0,32$ & \\
\hline Mai./2001 & $0,50 \pm 0,21$ & $0,22 \pm 0,46$ & $0,04 \pm 0,08$ & $0,76 \pm 0,52$ & \\
\hline Jun./2001 & $0,52 \pm 0,32$ & $0,17 \pm 0,49$ & $0,14 \pm 0,34$ & $0,83 \pm 0,64$ & \\
\hline Jul./2001 & $0,84 \pm 0,39$ & $0,15 \pm 0,27$ & $0,17 \pm 0,27$ & $1,16 \pm 0,57$ & \\
\hline Ago./2001 & $0,92 \pm 0,40$ & $0,07 \pm 0,06$ & $0,09 \pm 0,19$ & $1,08 \pm$ & \\
\hline Set./2001 & $0,73 \pm 0,28$ & $0,17 \pm 0,27$ & $0,14 \pm 0,25$ & $1,04=$ & \\
\hline Dez./2001 & $0,30 \pm 0,01$ & $0,09 \pm 0,03$ & $0,06 \pm 0,03$ & $0,45 \pm 0,04$ & \\
\hline Jan./2002 & $0,44 \pm 0,30$ & $0,03 \pm 0,12$ & $0,02 \pm 0,06$ & $0,40 \pm 0,32$ & $9,43 \pm 3,30$ \\
\hline Fev./2002 & $0,39 \pm 0,23$ & $0,08 \pm$ & $0,03 \pm C$ & $0,48 \pm$ & $9,16 \pm 3,99$ \\
\hline Mar./2002 & $0,35 \pm 0,13$ & $0,18 \pm 0,23$ & $0,04 \pm 0$ & $0,58 \pm 0,35$ & $11,22 \pm 3,17$ \\
\hline Abr./2002 & $0,39 \pm 0,21$ & $0,08 \pm 0,16$ & $0,02 \pm 0,03$ & $0,48 \pm 0,30$ & $8,79 \pm 2,58$ \\
\hline Mai./2002 & $0,55 \pm 0,61$ & $0,09 \pm 0,15$ & $0,01 \pm 0,03$ & $0,67 \pm 0,70$ & \\
\hline Jun./2002 & $0,77 \pm 0,45$ & $0,11 \pm 0,17$ & $0,02 \pm 0,05$ & $0,90 \pm 0,53$ & \\
\hline Jul./2002 & $1,30 \pm 0,75$ & $0,14 \pm 0,18$ & $0,02 \pm 0,04$ & $1,53 \pm$ & \\
\hline Ago./2002 & $0,11 \pm 0,08$ & $0,01 \pm 0,06$ & & $0,21 \pm 0,10$ & $7,34 \pm 1,69$ \\
\hline Set./2002 & $0,58 \pm 0,43$ & $0,05 \pm 0,13$ & & $0,59 \pm 0,46$ & $7,33 \pm 2,09$ \\
\hline Out./2002 & $0,79 \pm 0,61$ & & & & \\
\hline \multicolumn{6}{|l|}{ Nov./2002 } \\
\hline Dez./2002 & $0,18 \pm 0,14$ & $0,01 \pm 0,01$ & & $0,01 \pm 0,01$ & $0,20 \pm 1,74$ \\
\hline Jan./2003 & $0,13 \pm 0,09$ & $0,05 \pm 0,06$ & $0,03 \pm 0,07$ & $0,21 \pm 0,16$ & $6,80 \pm 3,14$ \\
\hline Fev./2003 & $0,47 \pm 0,16$ & $0,25 \pm 0,43$ & $0,13 \pm 0,18$ & $0,86 \pm 0,55$ & $7,92 \pm 3,70$ \\
\hline Mar./2003 & $0,06 \pm 0,03$ & $0,01 \pm 0,01$ & $0,01 \pm 0,03$ & $0,08 \pm 0,06$ & \\
\hline Abr./2003 & $0,34 \pm 0,22$ & $0,10 \pm 0,21$ & $0,04 \pm 0,09$ & $0,48 \pm 0,42$ & $6,75 \pm 2,83$ \\
\hline Mai./2003 & $0,45 \pm 0,29$ & $0,05 \pm 0,06$ & $0,04 \pm 0,07$ & $0,54 \pm 0,37$ & \\
\hline Jun./2003 & $0,40 \pm 0,18$ & $0,02 \pm 0,05$ & $0,04 \pm 0,10$ & $0,46 \pm 0,22$ & \\
\hline Jul./2003 & $0,79 \pm 0,54$ & $0,04 \pm 0,07$ & $0,01 \pm 0,04$ & $0,85 \pm 0,55$ & $6,34 \pm 2,29$ \\
\hline Ago./2003 & $0,93 \pm 0,49$ & $0,06 \pm 0,14$ & $0,01 \pm 0,05$ & $1,00 \pm 0,51$ & $4,82 \pm 2,41$ \\
\hline Set./2003 & $0,76 \pm 0,63$ & $0,05 \pm 0,15$ & $0,37 \pm 1,03$ & $1,18 \pm 1,31$ & $3,78 \pm 1,23$ \\
\hline Out./2003 & $0,57 \pm 0,55$ & $0,32 \pm 0,64$ & $0,09 \pm 0,17$ & $0,98 \pm 1,11$ & \\
\hline Nov./2003 & $0,41 \pm 0,25$ & $0,13 \pm 0,20$ & $0,16 \pm 0,22$ & $0,71 \pm 0,39$ & \\
\hline Dez./2003 & $0,08 \pm 0,03$ & $0,02 \pm 0,03$ & $0,05 \pm 0,12$ & $0,15 \pm 0,14$ & $3,19 \pm 1,90$ \\
\hline
\end{tabular}

marítimo (Pinus pinaster Aiton) (14,20 t ha-1) (Kavvadias et al., 2001); floresta subtropical úmida de 7 e 16 anos, com produção de 11,90 e $17,49 \mathrm{t} \mathrm{ha}^{-1} \mathrm{ano}^{-1}$, respectivamente (Arunachalam et al., 1998).

A composição de serrapilheira mostrou-se em 60 a 93\% de folhas, 5 a 32\% de galhos, 0 a $6 \%$ de flores e 3 a $17 \%$ de frutos, ressaltando-se que as folhas são o seu componente principal e sua fração depende, sobretudo, do cultivo formado, da estrutura do local e da idade das árvores (Zimmermann et al.,

A.

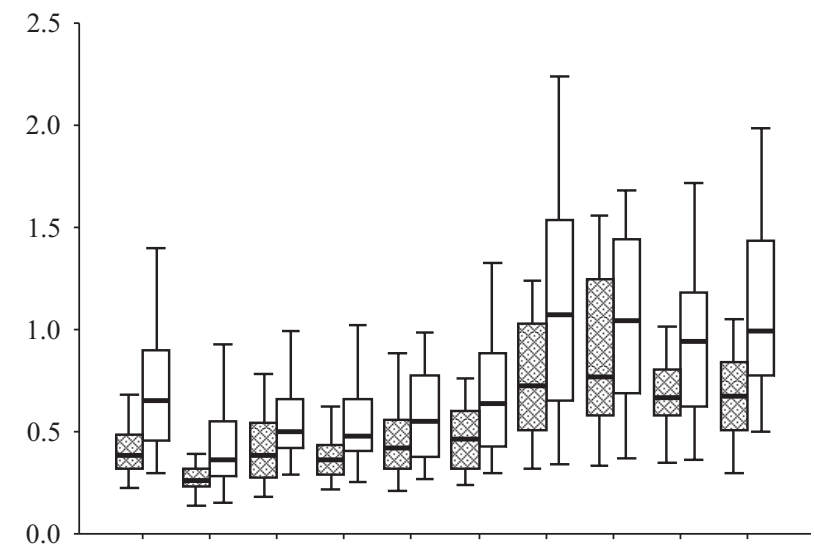

B.

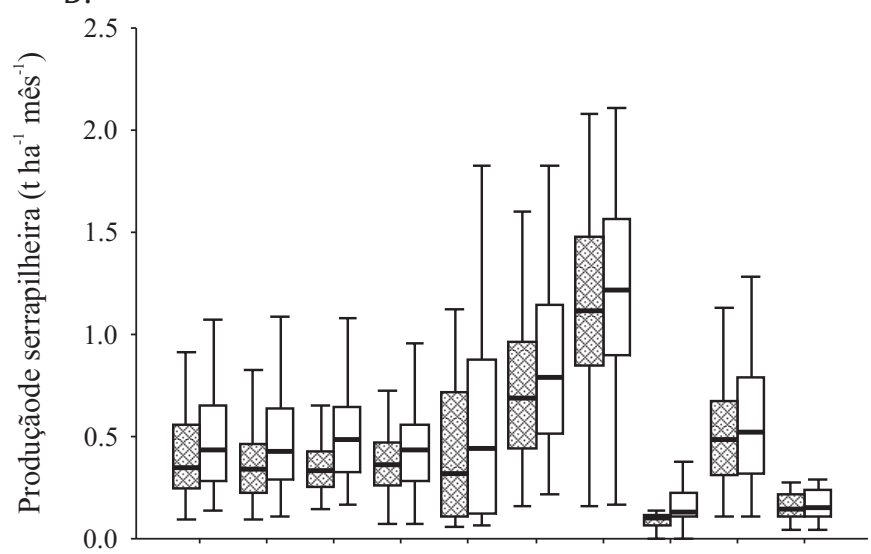

C.

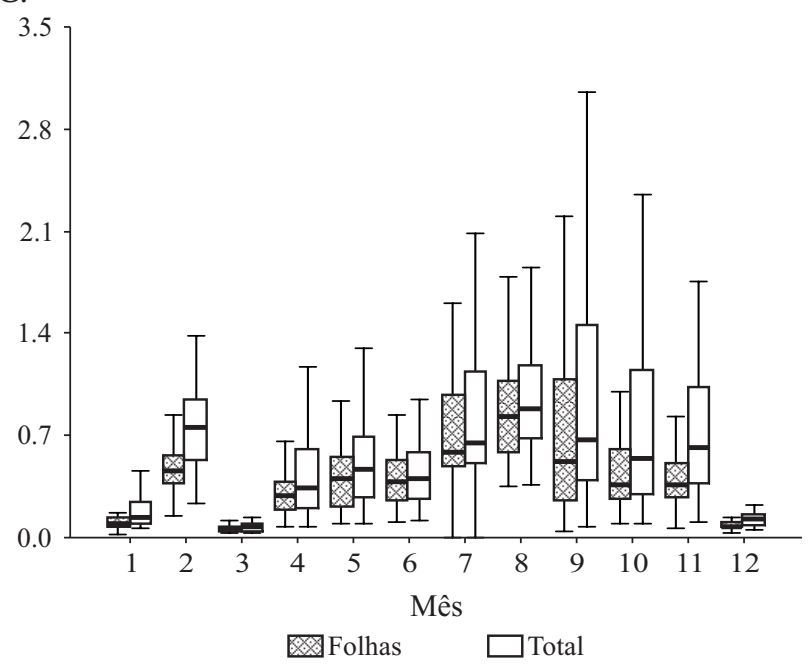

Figura 3. Produção de serrapilheira $\left(\mathrm{t} h \mathrm{a}^{-1} \mathrm{mês}^{-1}\right)$ em floresta de transição Amazônia Cerrado, em (A) 2001, (B) 2002 e (C) 2003 
2002). Apesar da variação sazonal da produção de serrapilheira, não se observou variação porcentual dos componentes de serrapilheira durante as diferentes estações.

A variação da produção de serrapilheira anual pode ser reportada em um intervalo de taxas de 1:1,32:1,13 para os anos 2001:2002:2003, respectivamente, indicando que a produção de 2002 foi superior a 2001 e 2003; esses valores de taxas foram concordantes com os valores definidos por Arunachalam et al. (1998).

A deposição de flores, mesmo menor que a de folhas, ocorreu somente nos meses de dezembro e janeiro (estação úmida) e a deposição de frutos não apresentou tendência com relação às condições climáticas, indicando que sua deposição seja possivelmente dependente da estratégia de dispersão adotada pela espécie para sua reprodução e não esteja em função das variações do microclima.

A análise de correlação entre produção de serrapilheira, temperatura, umidade relativa do ar e precipitação, apresentou correlações negativas significativas entre produção de serrapilheira e umidade relativa do ar $(r=-0,51)$ e precipitação $(r=-0,53)$ indicando que houve maior deposição mensal na estação seca que na estação úmida; não se verificou, porém, correlação significativa entre a produção de serrapilheira e a temperatura média mensal do ar.

\section{Acúmulo de serrapilheira no solo}

A massa média de serrapilheira acumulada no solo variou de 3,20 a 7,91 tha-1 em 2002 e 5,72 a 9,43 t ha-1 em 2003 (Tabela 1, Figura 4). Os valores apresentados em 2003 foram concordantes com os indicados em florestas naturais intactas de pinheiro marítimo (Pinus pinaster Aiton) (Kavvadias et al., 2001), e em floresta tropical (4,80 a 10,05 t ha-1 estação seca e 4,31 a 8,92 t ha-1 estação úmida) (Wieder \& Wright, 1995), além de mais elevados que os resultados mostrados para floresta subtropical (2,9 a 5,4 t ha-1) (Lodhiyal \& Lodhiyal, 2003). A composição do acúmulo de serrapilheira foi majoritariamente formada por folhas com valores médios de 69 a 78\%, em 2002, e 73 a 85\% em 2003, seguida de galhos, raízes e miscelânea (frutos e flores).

\section{Decomposição de serrapilheira}

A decomposição de serrapilheira por períodos como constante de decaimento (K) (Wieder \& Wright, 1995) estimada, variou de 0 a 0,044 por dia (0 a 16,06 ano-1), em 2002 e 2003 (Tabela 2). O coeficiente de retorno de serrapilheira $\left(\mathrm{K}_{\mathrm{L}}\right)$ foi 0,87 e 1,35 ano $^{-1}$ em 2002 e 2003, respectivamente, cujos valores foram similares aos encontrados em florestas tropicais primárias e secundárias (Proctor, 1987). O tempo de retorno (t) foi 1,15 e 0,75 ano $^{-1}$ em 2002 e 2003, respectivamente, valores inferiores aos constatados em floresta subtropical (1,26 a 1,33 ano $^{-1}$ ) (Lodhiyal \& Lodhiyal, 2003). Os valores de $\mathrm{K}^{\prime}$ foram de 0,47 e 0,57 $\mathrm{ano}^{-1}$, para 2002 e 2003, respectivamente, além de concordantes com os valores encontrados em floresta de pinheiro $\left(0,50\right.$ e 0,48 ano $\left.^{-1}\right)(\mathrm{Xu} \&$ Hirata, 2002).

Uma das características da floresta tropical e subtropical úmida é a produção de serrapilheira, com máximos valores na estação seca, sendo a folha a fração mais importante da

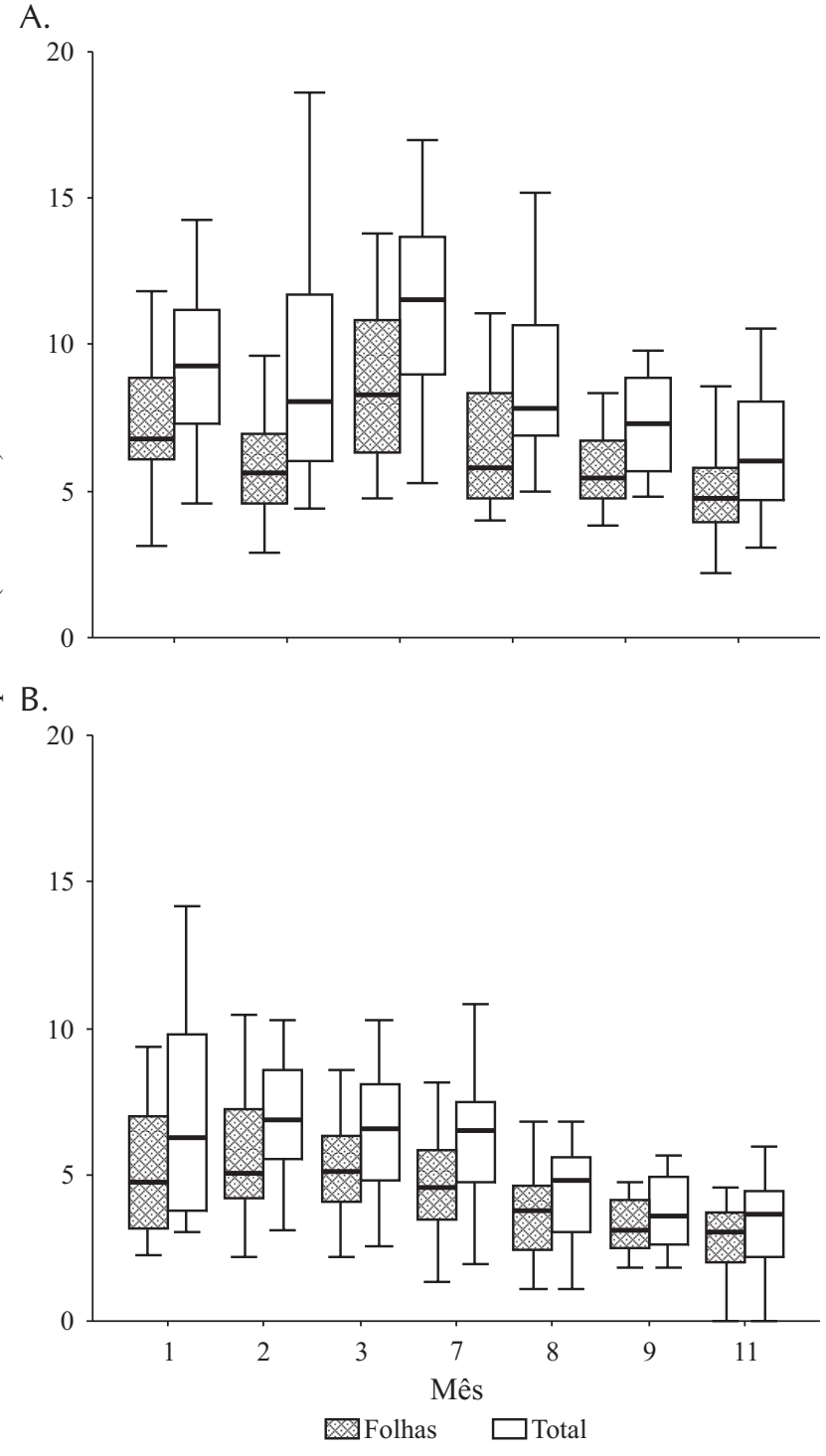

Figura 4. Acúmulo de serrapilheira sobre o solo em floresta de transição Amazônia Cerrado, em (A) 2002 e (B) 2003

serrapilheira (Arunachalam et al., 1998; Barajas-Guzmán \& Alvarez-Sánchez, 2003).

Golley (1978) menciona que a produção de serrapilheira em regiões tropicais e subtropicais ocorre na transição entre a estação seca e a chuvosa. No presente trabalho a maior produção de serrapilheira no ano 2001 ocorreu na estação seca e nos anos 2002 e 2003, no período de transição secoúmido. Esta maior produção se deve ao estresse hídrico, que

Tabela 2. Estimativa da constante de decaimento (K)

\begin{tabular}{lcc}
\hline Mês/Ano & \multicolumn{2}{c}{$\mathbf{K}\left(\mathbf{d i a}^{-1}\right)$} \\
Fevereiro & $\mathbf{2 0 0 2}$ & $\mathbf{2 0 0 3}$ \\
Abril & 0,0099 & \\
Maio & 0,0108 & 0,068 \\
Agosto & & 0,009 \\
Setembro & 0,028 \\
Novembro & & 0,044 \\
\hline
\end{tabular}


produz economia de água no ecossistema, resultando em uma perda de biomassa vegetal.

Com o retorno da precipitação ocorreu aumento na biodiversidade dos organismos decompositores do solo, aumentando a velocidade de decomposição da serrapilheira.

Estudos em invertebrados do solo mostram que, em estações secas, os movimentos da fauna do solo são mais profundos (Prieto et al., 1999). Os artrópodes, por exemplo, decompositores responsáveis por cerca de $20 \%$ da folhagem anual, em uma floresta tropical úmida com padrão irregular de chuvas, tem sua população diminuída durante períodos secos, quando comparados com períodos chuvosos.

\section{Decomposição de folhas}

Para determinação da perda de massa seca utilizaramse bolsas de nylon em dois experimentos instalados em distintos períodos do ano, ou seja, período úmido-seco e período seco-úmido. A perda de média de peso do material no período úmido-seco foi de $28,1 \%$ e, no período seco-úmido, de 23,2\%, em 91 e 129 dias, respectivamente (Figura 5). Obtiveram-se, em ambos os experimentos, correlações satisfatórias entre o tempo de exposição do material e o percentual de material remanescente na área em estudo, sendo os modelos da velocidade de decomposição em período seco-úmido e úmido-seco descritos por $\mathrm{y}=150,66 \mathrm{e}^{-0,0033 \mathrm{x}}$ $\left(R^{2}=0,9009\right)$ e $y=145,87 e^{-0,0017 x}\left(R^{2}=0,9223\right)$, respectivamente.

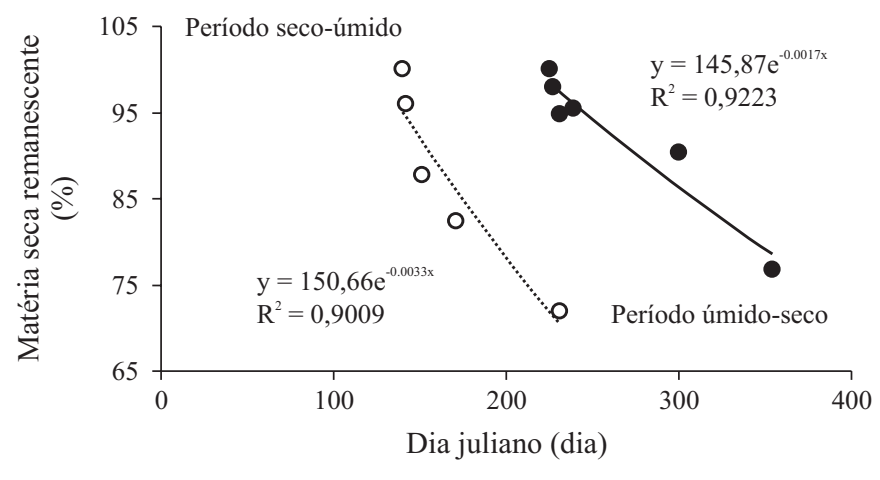

$\overline{\text { Figura 5. Decomposição de folhas nos períodos úmido-seco e seco-úmido }}$

Em termos de biomassa, as folhas de serrapilheira representam parcela substancial da produção de serrapilheira no solo, em virtude da sua concentração de nutrientes relativamente mais elevada que os demais componentes; além disso, a taxa de decomposição das folhas é mais elevada que a dos galhos e outros materiais, nos primeiros meses, em função dos principais componentes das folhas serem carboidratos solúveis de maior velocidade de decomposição que os demais componentes (Songwe et al., 1995). Apresentaramse, no presente estudo, modelos de velocidade de decomposição que indicam rápida decomposição do material em curtos intervalos de tempo (91 e 129 dias).

Ao se comparar os valores médios acrescidos dos respectivos desvios padrões para os dois períodos estudados, observa-se que a velocidade de decomposição foi maior no pe- ríodo úmido-seco. Estudos indicam que a decomposição é influenciada pela fauna do solo de cada região e pelas espécies formadoras da serrapilheira (Heneghan et al., 1998) e, ainda, que a qualidade da serrapilheira justifica mais as variações na decomposição que as condições ambientais (Xuluc-Tolosa et al., 2003) e a quantidade de serrapilheira (Warren \& Zou, 2002). O uso da correlação-regressão para determinar a taxa de decomposição no presente estudo foi um bom indicador para o modelo regional; entretanto, Heneghan et al. (1998) comentam que o uso de correlação-regressão não esclarece a importância da fauna nos processos de decomposição de serrapilheira.

\section{CONCLUSÕES}

1. A produção de serrapilheira apresentou-se de forma sazonal, com forte influência da umidade relativa do ar e precipitação, em uma floresta de transição.

2. As taxas de decomposição, calculadas por diferentes modelos, forneceram valores concordantes a estudos realizados em florestas tropicais úmidas.

3. A produção de serrapilheira foi majoritariamente composta de folhas, seguidas de galhos e miscelânea.

4. A decomposição de folhas em floresta de transição foi acelerada em ambos os períodos, seco-úmido e úmido-seco, sendo a velocidade de decomposição maior no período úmido-seco.

\section{LITERATURA CITADA}

Aerts, R. Climate, leaf litter chemistry and leaf litter decomposition in terrestrial ecosystems: A triangular relationship. Oikos, v.79, p.439-449, 1997.

Arunachalam, A.; Arunachalam, K. M.; Pandey, H. N.; Tripathi, R. S. Fine litterfall and nutrient dynamics during forest regrowth in the humid subtropics of north-eastern India. Forest Ecology and Management, v.110, p.209-219, 1998.

Barajas-Guzmán, G.; Alvarez-Sánchez, J. The relationships between litter fauna and rates of litter decomposition in a tropical rain forest. Applied Soil Ecology, v.24, p.91-100, 2003.

Cornu, S.; Luizão, F.; Rouiller, J.; Lucas, Y. Comparative study of litter decomposition and mineral element release in two Amazonian Forest ecosystems: Litter bag experiments. Pedobiologia, v.41, p.456-471, 1997.

Corrêa Neto, T. A.; Pereira, M. G.; Corrêa, M. E. F.; Anjos, L. H. C. Deposição de serrapilheira e mesofauna edáfica em áreas de eucalipto e floresta secundária. Floresta e Ambiente, v.8, n.1, p.70-75, 2001.

Coûteaux, M. M.; Bottner, P.; Berg. B. Litter decomposition, climate and litter quality. Tree, v.10, n.2, p.63-66, 1995.

Golley, F. B. Ciclagem de nutrientes em um ecossistema de floresta tropical úmida. 10.ed., São Paulo: USP, 1978. 256p.

Heneghan, L.; Coleman, D. C.; Zou, X.; Crossley Jr, D. A.; Haines, B. L. Soil microarthropod community structure and litter decomposition dynamics: A study of tropical and temperature sites. Applied Soil Ecology, v.9, p.33-38, 1998. 
Heneghan, L; Coleman, D. C.; Zou, X.; Crossley Jr.; D. A., Haines, B. L. Soil microarthropod contributions to decomposition dynamics: Tropical-temperate comparisons of a single substrate. Ecology, v.80, p.1873-1882, 1999.

Kavvadias, V. A.; Alifragis, D.; Tsiontsis, A.; Brofas, G.; Stamatelos, G. Litterfall, litter acumulation and litter decomposition rates in four forest ecosystems in northern Greece. Forest Ecology and Management, v.144, p.113-127, 2001.

Lamb, R. J. Litter fall and nutrient turnover in two eucalypt woodlands. Australian Journal of Botany, v.33, p.1-14, 1985.

Lavelle, P.; Blanchart, E.; Martin, A.; Martin, S.; Spain, A.; Toutan, F.; Barois, I.; Schaefer, R. A hierarchical model for decomposition in terrestrial ecosystems: Application to soils of the humid tropics, Biotropica, v.25, p.130-150, 1993.

Lodhiyal, N.; Lodhiyal, L. S. Biomass and net primary productivity of Bhabar Shisham forests in central Himalaya, India. Forest Ecology and Management, v.176, p.217-235, 2003.

Prieto, T. D.; Vázquez, G. M. M.; Rodríguez, A. C. Comunidades de la mesofauna edáfica en una selva inundable de la Reserva de la Biosfera de Sian Kaan, Quintana Roo, México. Revista de Biologia Tropical, v.47, p.489-492, 1999.

Proctor, J. Nutrient cycling in primary and old secondary rainforests. Applied Geography, v.7, p.135-152, 1987.

Reiners, W. A.; Reiners, N. M. Energy and nutrient dynamics of forest floor in three Minnesota forest. Journal Ecology, v.74, p.37-49, 1970.
Smith, V. C.; Bradford, M. A. Litter quality impacts on grassland litter decomposition are differently dependent on soil fauna across time. Applied Soil Ecology, v.24, p.197-203, 2003.

Songwe, N.; Okali, D. U.; Fasehum, F. Litter decomposition and nutrient release in a tropical rainforest, Southern Bakundu Forest Reserve, Cameroon. Journal Tropical Ecology, v.11, p.333-350, 1995.

Waring, R. H.; Schlesinger, W. H. Forest ecosystems: Concepts and management. St Louis: Academic Press, 1985. 340p.

Warren, M. W.; Zou, X. Soil macrofauna and litter nutrients in three tropical tree plantations on a disturbed site in Puerto Rico. Forest Ecology and Management, v.170, p.161-171, 2002.

Wieder, K.; Wright, J. S. Tropical forest litter dynamics and dry season irrigation on Barro Colorado island, Panama. Ecology, v.76, n.6, p.1971-1979, 1995.

Xu, X. N.; Hirata, E. Forest floor mass and litterfall in Pinus luchuensis plantations with and without broad-leaved trees. Forest Ecology and Management, v.157, p.165-173, 2002.

Xuluc-Tolosa, F. J.; Vester, H. F. M.; Ramírez-Marcial, N.; Castellanos-Albores, J.; Lawrence, D. Leaf litter decomposition of tree species in three successional phases of tropical dry secondary Forest in Campeche, México. Forest Ecology and Management, v.174, p.401-412, 2003.

Zimmermann, S.; Braun, S.; Conedera, M.; Blaser, P. Macronutrient inputs by litterfall as opposed to atmospheric deposition into two contrasting chestnut forest stands in southern Switzerland. Forest Ecology and Management, v.161, p.289-302, 2002. 